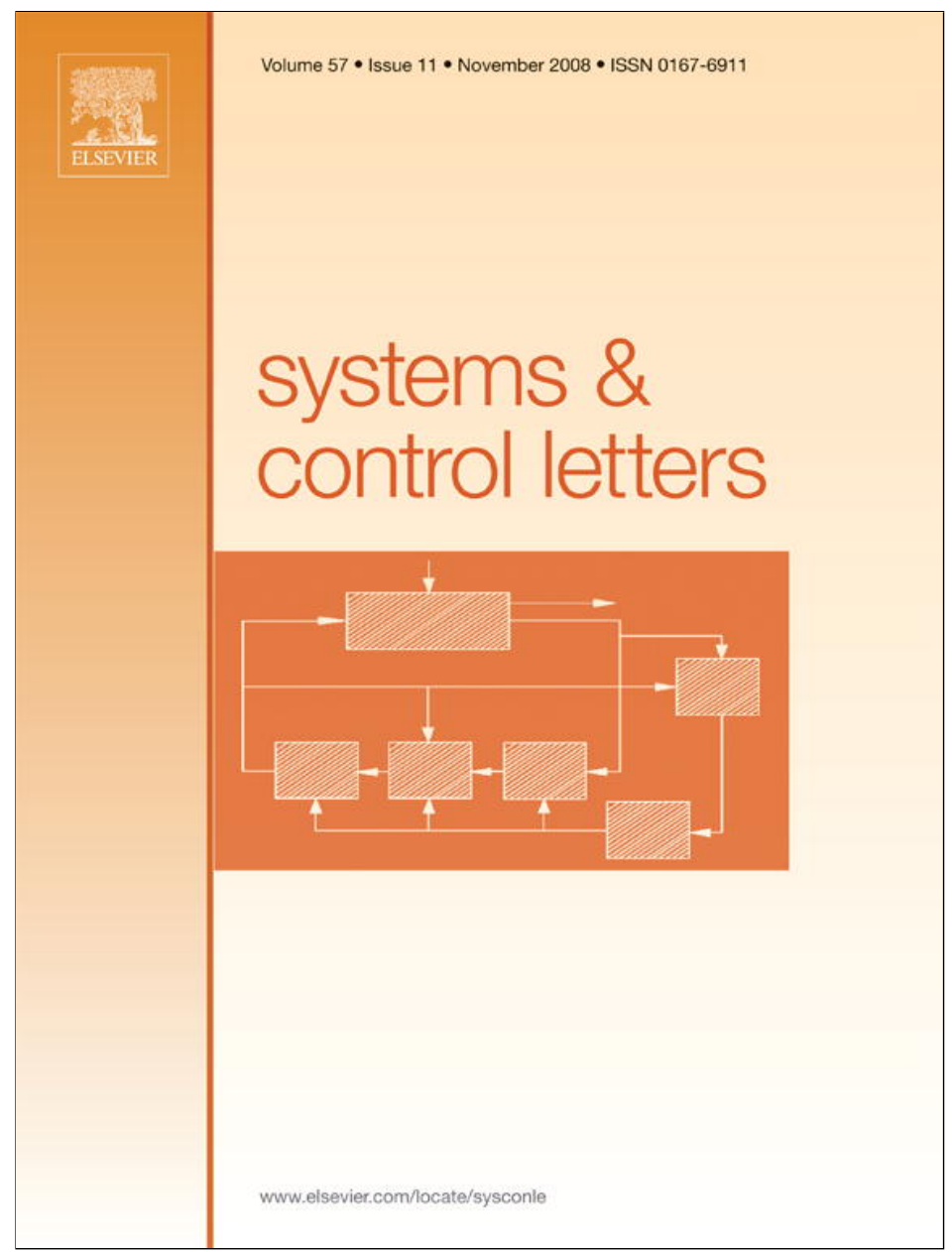

This article appeared in a journal published by Elsevier. The attached copy is furnished to the author for internal non-commercial research and education use, including for instruction at the authors institution and sharing with colleagues.

Other uses, including reproduction and distribution, or selling or licensing copies, or posting to personal, institutional or third party websites are prohibited.

In most cases authors are permitted to post their version of the article (e.g. in Word or Tex form) to their personal website or institutional repository. Authors requiring further information regarding Elsevier's archiving and manuscript policies are encouraged to visit:

http://www.elsevier.com/copyright 


\title{
Optimal control of wave linear repetitive processes
}

\author{
Michael Dymkov ${ }^{a}$, Eric Rogers ${ }^{\mathrm{b}, *}$, Krzysztof Galkowski $^{\mathrm{c}}$, Siarhei Dymkou ${ }^{\mathrm{d}}$ \\ a Department of Mathematics, Belarus State Economic University, Partizanskiy Ave 26, 220070 Minsk, Belarus \\ ${ }^{\mathrm{b}}$ School of Electronics and Computer Science, University of Southampton, Southampton SO17 1BJ, UK \\ ${ }^{c}$ Institute of Control and Computation Engineering, University of Zielona Gora, Poland \\ ${ }^{\mathrm{d}}$ Department of Applied Mathematics II, Friedrich-Alexander-University of Erlangen-Nuremberg, Martensstra-e 3, 91058 Erlangen, Germany
}

\section{A R T I C L E I N F O}

\section{Article history:}

Received 9 August 2007

Received in revised form

24 May 2008

Accepted 24 May 2008

Available online 2 July 2008

\section{Keywords:}

Repetitive dynamics

Optimal control

\begin{abstract}
A B S T R A C T
This paper gives new results on optimal control of the so-called wave discrete linear repetitive processes which find novel application in the modelling of physical examples. These processes have dynamics which are not restricted to the upper right quadrant of the 2D plane and hence the current control results for repetitive processes or $2 \mathrm{D}$ systems are not applicable.
\end{abstract}

(c) 2008 Elsevier B.V. All rights reserved.

\section{Introduction}

The unique characteristic of a repetitive process is a series of sweeps, termed passes, through a set of dynamics defined over a fixed finite duration known as the pass length. On each pass an output, termed the pass profile, is produced which acts as a forcing function on, and hence contributes to, the dynamics of the next pass profile. This, in turn, leads to the unique control problem for these processes in that the output sequence of pass profiles generated can contain oscillations that increase in amplitude in the pass-to-pass direction.

Physical examples of repetitive processes include long-wall coal cutting and metal rolling operations. Also in recent years applications have arisen where adopting a repetitive process setting for analysis has distinct advantages over alternatives. For the details on all these examples see [1] and the relevant references in this research monograph.

In this paper, we introduce the so-called wave repetitive processes, using as motivation the discretization of physical systems whose dynamics are governed by partial differential equations. The dynamics of these processes are defined over the upper-half, as opposed to a restricted upper right quarter, of the $2 \mathrm{D}$ plane in the previous work. This means that the existing control theory for repetitive processes is not applicable and in this paper we formulate and solve an optimal control problem for the wave model

\footnotetext{
* Corresponding author.

E-mail address: etar@ecs.soton.ac.uk (E. Rogers).
}

case using the operator setting in the relevant infinite-dimensional spaces. In effect, the results are obtained by first constructing a standard, or 1D, equivalent model description of the dynamics in such spaces.

\section{Background}

The unique feature of repetitive processes is that the dynamics evolve over the finite pass length, resetting then occurs and as the next pass evolves there is an explicit contribution from the output, or pass profile, produced on the previous pass. This interaction is the source of the unique control problem, i.e. oscillations in the output (pass profile) sequence which can increase in amplitude in the pass-to-pass direction.

The currently available theory for these processes only covers one sub-class and, in particular, those which evolve over the restricted quadrant of the $2 \mathrm{D}$ plane. Let $m$ denote the along the pass variable, $N$ the finite pass length, and $t$ the pass number. Then the domain of these variables for the processes considered so far is $\{(t, m): t \geq 0,0 \leq m \leq N\}$.

In fact, there are examples where a model over this domain cannot be used to capture the dynamics of a repetitive process. Consider, for example, a system described by the spatio-temporal partial differential equation

$$
\frac{\partial x(\sigma, \tau)}{\partial \sigma}=A_{1} \frac{\partial^{2} x(\sigma, \tau)}{\partial \tau^{2}}+A_{2} x(\sigma, \tau)+B u(\sigma, \tau)
$$

where $\sigma$ is the temporal variable, $\tau$ is the spatial variable, $u(\sigma, \tau)$ is the control input, and $x(\sigma, t)$ the system output. For computational 
purposes one approach is to discretize the partial differential equation where here the resulting discrete variables are denoted by $t$ and $m$ respectively. Suppose, for example, that the following approximations are used

$$
\left(\frac{\partial x}{\partial \sigma}\right)_{t, m}=\frac{1}{\Delta t}(x(t+1, m)-x(t, m))
$$

and

$$
\left(\frac{\partial^{2} x}{\partial \tau^{2}}\right)_{t, m}=\frac{1}{\Delta \tau^{2}}(x(t, m+1)-2 x(t, m)+x(t, m-1))
$$

where $\Delta \tau$ and $\Delta t$ are the corresponding discretization periods.

The approximate process dynamics can now be treated as a special case of

$x(t+1, m)=\sum_{i=-N}^{N} A_{i} x(t, m+i)+B u(t, m)$

$t=0,1 \ldots$ where $N$ is a positive integer, $x(t, m) \in \mathbb{R}^{n}, u(t, m) \in$ $\mathbb{R}^{r}$ with the given boundary conditions

$x(0, m)=\phi(m), \quad m \in[-N, N]$

for any $(t, m)$, where if the spatial domain is unbounded then $m \in[-\infty, \infty]$. Now if we interpret $t$ and $m$ as the pass-to-pass and along the pass variables respectively we have a so-called wave repetitive process.

The model structure is substantially different in structure from the discrete linear repetitive processes considered in, for example, [1] whose domain of operation is the restricted positive quadrant of the 2D plane defined by $\{(t, m): t \geq 0,0 \leq m \leq N\}$. (Note also that similar approaches to modelling flexible distributed parameter systems for control analysis can be found in [2] and the relevant references cited in this thesis.) This means we cannot apply existing linear repetitive process theory nor that for other quarter plane 2D systems, e.g. [3].

With the overall aim of moving to a theory for control design for wave repetitive processes, this paper develops a 1D equivalent model for the process dynamics and then solves an optimal control problem which is also shown to be expressible in feedback form. The analysis here is in the spirit of [4] for optimal control of finitedimensional 1D linear systems.

\section{Optimization analysis}

For analysis purposes, we can treat the case of $N=\infty$ and then obtain the results for any finite $N$ by projection. Moreover, in practical applications only a finite number of passes, say $T$, will actually be completed. Hence we begin by considering the optimal control/optimization problem: find the admissible control vector $u^{0}(t, m)$ which minimizes the cost function

$J(u)=\sum_{t=0}^{T} \sum_{m=-\infty}^{\infty}\langle Q x(t, m), x(t, m)\rangle+\langle R u(t, m), u(t, m)\rangle$

over the solutions of (4) and (5), with $N=\infty$ and $\langle\cdot, \cdot\rangle$ denotes the inner product (on the corresponding function spaces). Also it is assumed that the matrix $Q$ is symmetric positive semi-definite, written $Q \geq 0$, the matrix $R$ is symmetric positive-definite, written $R>0$, and the matrices $A_{i}$ satisfy

$$
\sum_{i=-\infty}^{+\infty}(1+\varepsilon)^{i}\left\|A_{i}\right\|<\infty
$$

for some real number $\varepsilon>0$, where $\|\cdot\|$ is the induced norm. This last assumption ensures that the series $\sum_{i=-\infty}^{+\infty} z^{i} A_{i}$ converges in a domain which includes the unit disc of complex plane $\mathbb{C}$. (In physical terms this cost function is the sum of quadratic terms in the pass profile and control vectors respectively summed over all passes completed.)

By way of notation we let $l^{2}\left(\mathbb{R}^{n}\right)$ and $l^{2}\left(\mathbb{R}^{r}\right)$ denote the spaces of the square summable sequences in $\mathbb{R}^{n}$ and $\mathbb{R}^{r}$ respectively. Also, introduce (where $N=\infty$ )

$y=\left\{y_{t}, t=0,1, \ldots, T\right\}, \quad y \in\left(l^{2}\left(\mathbb{R}^{n}\right)\right)^{T+1}$,

$u=\left\{u_{t}, t=0,1, \ldots, T\right\}, \quad u \in\left(l^{2}\left(\mathbb{R}^{r}\right)\right)^{T+1}$

where (over $\mathbb{Z}$ )

$y_{t}=\{\ldots, x(t,-1), x(t, 0), x(t, 1), \ldots\} \in l^{2}\left(\mathbb{R}^{n}\right)$

$u_{t}=\{\ldots, u(t,-1), u(t, 0), u(t, 1), \ldots\} \in l^{2}\left(\mathbb{R}^{r}\right) \quad \forall t$

$\phi=\{\ldots, \phi(-1), \phi(0), \phi(1), \ldots\} \in l^{2}\left(\mathbb{R}^{n}\right)$.

Then it is straightforward to show that the optimization problem defined by (4), (5) and (6) can be re-written in operator form as

$y=L u+w, \quad w=\left\{\phi, \mathcal{A} \phi, \ldots,(\mathcal{A})^{\mathrm{T}} \phi\right\}$

with cost function

$J(u)=\left\langle\left(\mathcal{R}+L^{*} \mathcal{Q} L\right) u, u\right\rangle+2\left\langle L^{*} \mathcal{Q} w, u\right\rangle+\langle\mathcal{Q} w, w\rangle$.

Hence a unique optimal solution $u^{0} \in l^{2}\left(\mathbb{R}^{r}\right)$ if it exists can be presented also in the operator form as

$u^{0}=-\left(\mathcal{R}+L^{*} \mathcal{Q} L\right)^{-1} L^{*} \mathcal{Q} \phi$

where $L:\left(l^{2}\left(\mathbb{R}^{r}\right)\right)^{T+1} \rightarrow\left(l^{2}\left(\mathbb{R}^{n}\right)\right)^{T+1}$ and $\mathcal{A}: l^{2}\left(\mathbb{R}^{n}\right) \rightarrow l^{2}\left(\mathbb{R}^{n}\right)$ are the operators defined by

$$
\begin{aligned}
(L \gamma)_{t}= & \mathscr{B} \gamma_{t-1}+\mathcal{A} \mathscr{B} \gamma_{t-2}+\cdots+\mathcal{A}^{t-1} \mathscr{B} \gamma_{0},(L \gamma)_{0}=0, \\
& t>0,
\end{aligned}
$$

and

$(\mathcal{A} \alpha)(m)=\sum_{i=-\infty}^{+\infty} A_{i} \alpha(m+i), \quad m \in \mathbb{Z}$

respectively, and the operators $\mathcal{B}, \mathcal{R}$, and $\mathcal{Q}$ are defined in an obvious way.

The adjoint operator $L^{*}$ is defined (as usual) by

$\left(L^{*} \beta\right)_{t}=\mathscr{B}^{*} \beta_{t+1}+\mathscr{B}^{*} \mathcal{A}^{*} \beta_{t+2}+\cdots+\mathscr{B}^{*} \mathcal{A}^{* T-t-1} \beta_{T}$

and the adjoint operator $\mathcal{A}^{*}: l^{2}\left(\mathbb{R}^{n}\right) \rightarrow l^{2}\left(\mathbb{R}^{n}\right)$ is

$\left(\mathcal{A}^{*}\right)(\psi)=\sum_{i=-\infty}^{+\infty} A_{i}^{*} \psi(m-i)$

where the $A_{i}^{*}$ is the complex conjugate transpose of $A_{i}$. Note also that since $\mathcal{Q} \geq 0, \mathcal{R}>0$ then the operator $\mathcal{R}+L^{*} \mathcal{Q} L$ is invertible.

The operator based solution (10) is not in a form suitable for actual implementation but it can be converted to such, starting from the following result.

Theorem 1. The boundary-value problem

$$
\begin{aligned}
& x(t+1, m)=\sum_{i=-\infty}^{\infty} A_{i} x(t, m+i)-B R^{-1} B^{*} z(t, m) \\
& z(t, m)=\sum_{i=-\infty}^{\infty} A_{i}^{*} z(t+1, m-i)+Q x(t+1, m) \\
& (t, m) \in\{0, \ldots, T\} \times \mathbb{Z}, \quad x(0, m)=\varphi(m), \\
& z(T, m)=0, \quad m \in \mathbb{Z}
\end{aligned}
$$

has a solution in $l^{2}\left(\mathbb{R}^{n}\right)$. 
Proof. Let $y_{t}, w_{t}$ be the elements of $l^{2}\left(\mathbb{R}^{n}\right)$ for which

$\left(y_{t}\right)(m)=x(t, m)$,

$\left(w_{t}\right)(m)=z(t, m), m \in \mathbb{Z}, \quad t \in\{0, \ldots, T\}$.

Then (14) can be rewritten in operator form as

$y_{t+1}=\mathcal{A} y_{t}-\mathscr{B} \mathcal{R}^{-1} \mathcal{B}^{*} w_{t}, \quad y_{0}=\varphi$

$w_{t}=\mathcal{A}^{*} w_{t+1}+\mathcal{Q} y_{t+1}, \quad w_{T}=0$.

Also with (4) rewritten in operator form set $y_{0}=\varphi$ and $u_{t}=u_{t}^{0}$, where $u^{0}=\left\{u_{0}^{0}, u_{1}^{0}, \ldots, u_{T}^{0}\right\}$ is defined by (10). Then we can determine $y_{t}^{0}, t \in\{0, \ldots, T\}$ from

$y_{t+1}=\mathcal{A} y_{t}+\mathscr{B} u_{t}=\mathcal{A} y_{t}-\mathscr{B}\left(\mathcal{R}+L^{*} \mathcal{Q} L\right)^{-1} L^{*} \mathcal{Q} w_{t}$,

$$
t \in\{0,1, \ldots, T\}
$$

as

$y_{t}^{0}=\mathcal{A}^{t} \varphi-\mathscr{B}\left(\mathcal{R}+L^{*} \mathcal{Q} L\right)^{-1} L^{*} \mathcal{Q} \sum_{i=0}^{t-1} \mathcal{A}^{i} w_{t-1-i}$.

Also the solution of the optimization problem considered here, i.e. $u^{0}=-\left(\mathcal{R}+L^{*} Q L\right)^{-1} L^{*} \mathcal{Q} \omega_{0}$ can be rewritten as $u^{0}=-\mathcal{R}^{-1} \mathscr{B}^{*} \omega_{0}$ and hence $y_{t}$ here can be written as

$y_{t}^{0}=\mathcal{A}^{t} \varphi-\sum_{i=0}^{t-1} \mathcal{A}^{i} \mathcal{B} \mathcal{R}^{-1} \mathcal{B}^{*} w_{t-1-i}, \quad t \in\{0, \ldots, T\}$.

Substituting this last result into the second equation in (15) and using the boundary condition $w_{T}=0$, gives

$w_{t}^{0}=\sum_{i=0}^{T-t} \mathcal{A}^{* i} Q y_{t+i}^{0}, \quad t \in\{0, \ldots, T\}$.

Hence

$w_{t}^{0}=\sum_{i=0}^{T-t} \mathcal{A}^{* i} Q y_{t+i}^{0}, \quad t \in\{0, \ldots, T\}$

and therefore the functions $\left(y_{t}^{0}, w_{t}^{0}\right), t \in\{0, \ldots, T\}$ satisfy the second equation in (15) and $y_{0}^{0}=\varphi, w_{T}^{0}=0$.

To complete the proof, we now require to show that

$u_{t}^{0}=-\mathcal{R}^{-1} \mathcal{B}^{*} w_{t}^{0}, \quad t \in\{0, \ldots, T\}$

where on multiplying both sides of (10) by $\left(\mathcal{R}+L^{*} \mathcal{Q} L\right)$ we have

$\left(\mathcal{R}+L^{*} \mathcal{Q} L\right) u^{0}=-\left(\mathcal{R}+L^{*} \mathcal{Q} L\right)\left(\mathcal{R}+L^{*} \mathcal{Q} L\right)^{-1} L^{*} \mathcal{Q} \omega$

and hence

$u^{0}=-\mathcal{R}^{-1} L^{*} Q y^{0}$.

Writing (17) in terms of the operator defined by (13) now gives

$\left(\mathscr{B}^{*} w^{0}\right)_{t}=\sum_{i=0}^{T-t-1} \mathcal{B}^{*} \mathcal{A}^{* i} \mathcal{Q} y_{t+i+1}^{0}=\left(L^{*} \mathcal{Q} y^{0}\right)_{t}, \quad t \in\{0, \ldots, T\}$.

Hence $u^{0}=-\mathcal{R}^{-1}\left(L^{*} Q y^{0}\right)=-\mathcal{R}^{-1} \mathcal{B}^{*} w^{0}$ and the proof is complete.

The following result now gives a solution to the optimal control problem considered here.

Theorem 2. The optimal control problem (4)-(6) has unique solution $u^{0}(t, m)=-R^{-1} B^{*} z(t, m), \quad t \in\{0, \ldots, T\}, m \in \mathbb{Z}$

where $z(t, s)$ is the solution of (14).
Proof. The uniqueness of the optimal control has already been established (see (10)). Let $(x(t, m), z(t, m)), t \in\{0, \ldots, T\}, m \in$ $\mathbb{Z}_{+}$, be a solution of the system (14), consider the function

$\hat{u}(t, m)=-R^{-1} B^{*} z(t, m), \quad t \in\{0, \ldots, T\}, m \in \mathbb{Z}_{+}$

and rewrite (14) (in operator form) as

$y_{t+1}=\mathcal{A} y_{t}-\mathcal{B} \mathcal{R}^{-1} \mathcal{B}^{*} w_{t}, \quad w_{t}=\mathcal{A}^{*} w_{t+1}+\mathcal{Q} y_{t+1}$,

$y_{0}=\varphi, \quad w_{T}=0$.

Then it follows immediately that

$y_{t}=\mathcal{A}^{t} \varphi-\sum_{i=0}^{t-1} \mathcal{A}^{i} \mathscr{B} \mathcal{R}^{-1} \mathcal{B}^{*} w_{t-1-i}, \quad t \in\{0, \ldots, T\}$,

$w_{t}=\sum_{i=0}^{T-t} \mathcal{A}^{* i} \mathcal{Q} y_{t+i}$

and

$\hat{v}_{t}=\mathcal{R}^{-1} \mathscr{B}^{*} w_{t}$

where we use $\hat{v}_{t}$ to denote $\left(\hat{v}_{t}\right)(m)=\hat{u}(t, m)$.

Using (19), $\hat{v}=\left(\hat{v}_{0}, \ldots, \hat{v}_{T}\right)$ can be written in the form

$\hat{v}=-\mathcal{R}^{-1} L^{*} Q y$.

Then

$\mathcal{R} \hat{v}=-L^{*} Q y$.

Conversely, from the first equation of (15) we have that $y=\omega-L \mathcal{R}^{-1} \mathscr{B}^{*} w$ or $y=\omega+L \hat{v}$

and therefore

$$
\begin{aligned}
\mathcal{R} \hat{v} & =-L^{*} \mathcal{Q} y+L^{*} \mathcal{Q} \omega-L^{*} \mathcal{Q},=L^{*} \mathcal{Q}(\omega-y)-L^{*} \mathcal{Q} \omega \\
& =-L^{*} \mathcal{Q} L \hat{v}-L^{*} \mathcal{Q} \omega \\
\mathcal{R} \hat{v} & =-L^{*} \mathcal{Q} y+L^{*} \mathcal{Q} \omega-L^{*} \mathcal{Q} \omega^{*} \mathcal{Q}(\omega-y)-L^{*} \mathcal{Q} \omega \\
& =-L^{*} \mathcal{Q} L \hat{v}-L^{*} \mathcal{Q} \omega
\end{aligned}
$$

and

$\hat{v}=-\left(\mathcal{R}+L^{*} \mathcal{Q} L\right)^{-1} L^{*} \mathcal{Q} \omega$.

Hence $\hat{v}$ coincides with $u^{0}$ defined by formula (10) and therefore $\hat{u}(t, m)=u^{0}(t, m)=-\mathcal{R}^{-1} \mathcal{B}^{*} z(t, m)$ as required.

\section{Optimal feedback control}

Here we seek a feedback solution of the optimal control problem. Consider the linear operators $P_{t}: l^{2}\left(\mathbb{R}^{n}\right) \rightarrow l^{2}\left(\mathbb{R}^{n}\right), t=$ $1, \ldots, T-1, P_{T}=0$ and also let $u^{0}$ be the optimal control for (4)-(6) and $x^{0}$ the corresponding trajectory generated by (4). Then optimal feedback control problem is to find linear operators $P_{t}, t \geq 0$, such that

$u_{t}^{0}=-\mathcal{R}^{-1} \mathscr{B}^{*} P_{t} x_{t}^{0}, \quad t=1, \ldots, T-1$.

We now have the following result.

Theorem 3. If the optimal feedback control problem has a solution then the operators $P_{t}$ satisfy

$P_{t-1}+\left(\mathcal{L}+\mathcal{A}^{*} P_{t}\right) \mathcal{B} \mathcal{R}^{-1} \mathcal{B}^{*} P_{t-1}=\left(\mathcal{L}+\mathcal{A}^{*} P_{t}\right) \mathcal{A}$,

$P_{T}=0, \quad t \geq 0$.

Moreover, the corresponding minimum value of the cost function, denoted by $J^{0}$, is given by $J^{0}=\left\langle P_{0} \varphi, \mathcal{A} \varphi\right\rangle$. 
Proof. Suppose that (23) holds. Then $x^{0}$ is such that

$y_{t+1}=\left(\mathcal{A}-\mathscr{B} \mathcal{R}^{-1} \mathcal{B}^{*} P_{t}\right) y_{t}, \quad t \in\{0, \ldots, T\}, y_{0}=\varphi$.

Now substitute $u^{0}=-\mathcal{R}^{-1} \mathcal{B}^{*} P_{t} y_{t}^{0}$ into (4) written in operator form to obtain

$y_{t+1}=\mathcal{A} y_{t}+\mathscr{B} u_{t}=\left(\mathcal{A}-\mathscr{B} \mathcal{R}^{-1} \mathcal{B}^{*} P_{t}\right) y_{t}$.

The solution of (25) can be written (where $s$ is an arbitrary index) as

$y_{t}^{0}=F_{t-1}\left(F_{t-2} \ldots\left(F_{s} y_{s}^{0}\right)\right) \quad \forall t>s \geq 0$

where $F_{t}=\mathcal{A}-\mathscr{B} \mathcal{R}^{-1} \mathscr{B}^{*} P_{t}$. Hence we have $y_{t+1}=F_{t} y_{t}$ with initial condition $y_{s}=y_{s}^{0}$. Noting (18) it is easy to show that

$L^{*}\left(\mathcal{Q} y^{0}\right)_{t}=\mathscr{B}^{*}\left(\mathcal{Q} y^{0}\right)_{t+1}+\cdots+\mathscr{B}^{*} \mathcal{A}^{*(T-t-1)}\left(\mathcal{Q} y^{0}\right)_{T}$

$=\mathscr{B}^{*}\left[\left(\mathcal{Q} y^{0}\right)_{t+1}+\mathcal{A}^{*}\left(\mathcal{Q} y^{0}\right)_{t+2}+\cdots+\mathcal{A}^{*(T-t-1)}\left(\mathcal{Q} y^{0}\right)_{T}\right]$.

Therefore

$-\mathcal{R}^{-1} \mathscr{B}^{*} P_{t} y_{t}^{0}=u_{t}^{0}=\left(-\mathcal{R}^{-1} L^{*}\left(y^{0}\right)_{t}\right.$

$=-\mathcal{R}^{-1} \mathscr{B}^{*}\left[\left(\mathcal{Q} y^{0}\right)_{t+1}+\cdots+\mathcal{A}^{*(T-t-1)}\left(\mathcal{Q} y^{0}\right)_{T}\right]$.

Using $y_{t+1}=F_{t} y_{t}$ and starting with an arbitrary index $s$ now yields $y_{t+1}^{0}=F_{t} y_{t}^{0}, \quad y_{t+2}^{0}=F_{t+1}\left(F_{t} y_{t}^{0}\right), \ldots, y_{T}^{0}=F_{T-1}\left(F_{T-2}\left(\cdots F_{t} y_{t}^{0}\right)\right)$ and therefore

$P_{t} y_{t}^{0}=\left(\mathcal{Q} F_{t}+\mathcal{A}^{*} \mathcal{Q} F_{t+1} F_{t}+\cdots+\mathcal{A}^{*(T-t-1)} \mathcal{Q} F_{T-1} F_{T-2} \cdots F_{t}\right) y_{t}$.

Hence the operators $P_{t}$ must satisfy

$P_{t-1}=\mathcal{Q} F_{t-1}+\mathcal{A}^{*} Q F_{t} F_{t-1}+\cdots+\mathcal{A}^{* T-t} \mathcal{Q} F_{T-1} \ldots F_{t-1}$

with $P_{T}=0$, or, in recurrent form,

$P_{t-1}+\left(\mathcal{Q}+\mathcal{A}^{*} P_{t}\right) \mathscr{B} \mathcal{R}^{-1} \mathscr{B}^{*} P_{t-1}=\left(\mathcal{Q}+\mathcal{A}^{*} P_{t}\right) \mathcal{A}$,

$t=1, \ldots, T, P_{T}=0$.

Let $P_{t}^{0}$ be a solution of (24). Then (after some routine manipulations)

$\left(P_{t-1}^{0} y_{t-1}^{0}, \mathcal{A} y_{t-1}^{0}\right)-\left(P_{t} y_{t}^{0}, \mathcal{A} y_{t}^{0}\right)=\left(\mathcal{R} v_{t-1}^{0}, v_{t-1}^{0}\right)+\left(\mathcal{Q} y_{t}^{0}, y_{t}^{0}\right)$

and then

$$
\begin{aligned}
J\left(v^{0}\right) & =\sum_{t=1}^{T}\left(\mathcal{Q} y_{t}^{0}, y_{t}^{0}\right)+\left\langle\mathcal{R} v_{t-1}^{0}, v_{t-1}^{0}\right\rangle \\
& =\sum_{t=1}^{T}\left[\left\langle P_{t-1}^{0} y_{t-1}^{0}, \mathcal{A} y_{t-1}^{0}\right\rangle-\left\langle P_{t}^{0} y_{t}^{0}, \mathcal{A} y_{t-1}^{0}\right\rangle\right]=\left\langle P_{0} y_{0}^{0}, \mathcal{A} y_{0}^{0}\right\rangle \\
& =\left\langle P_{0}^{0} \varphi, \mathcal{A} \varphi\right\rangle
\end{aligned}
$$

and the proof is complete.

\section{Optimal control for $T \rightarrow \infty$}

The pass length $T$ can take any finite value and hence in this section we consider the problem of the previous section for the case when $T \rightarrow \infty$. Let $l_{2}^{2}\left(\mathbb{R}^{n}\right)$ be the space of all the sequences $\{v(t, m)\},(t, m) \in \mathbb{Z}_{+} \times \mathbb{Z}$ of elements from $\mathbb{R}^{n}$ such that $\sum_{(t, m) \in \mathbb{Z}_{+} \times \mathbb{Z}}\|v(t, m)\|^{2}<\infty$. Assume also that the spectral radius of the operator defined by (12) satisfies $r(\mathcal{A})<1$. Then we have the following result.
Theorem 4. Assume $T \rightarrow \infty$ and suppose also that

$$
\begin{aligned}
& \sum_{i=-\infty}^{\infty}\left\|A_{i}\right\|+\left\|B R^{-1} B^{*}\right\|^{2}<1 \\
& \|Q\|<1-\left(\sum_{i=-\infty}^{\infty}\left\|A_{i}\right\|\right)^{2} /\left(1-\left\|B R^{-1} B^{*}\right\|^{2}\right) .
\end{aligned}
$$

Then the optimal control for (4) and (5) is given by

$u_{t}^{0}=-\mathscr{B}^{*} P x_{t}^{0}, \quad t>0$

where $x_{t}^{0}, t \in \mathbb{Z}_{+}$, is the unique solution of

$x_{t+1}=\left(\mathscr{A}-\mathscr{B} \mathscr{B}^{*} P\right) x_{t}, \quad x_{0}=\varphi$

and $P: l^{2}\left(\mathbb{R}^{n}\right) \rightarrow l^{2}\left(\mathbb{R}^{n}\right)$ is the bounded linear operator which satisfies

$P=\left(\mathcal{R}+\mathcal{A}^{*} P\right)\left(\mathcal{A}-\mathscr{B} \mathcal{B}^{*} P\right)$.

Also, the minimum cost value is $J^{0}=\langle P \varphi, \mathcal{A} \varphi\rangle$.

Proof. As before, it can be shown that the unique optimal control for this case exists and can be expressed in the operator form (10). Now let $N>1$ be a fixed integer and use $P_{t}, t=0,1, \ldots, N$ to denote the solutions of (24). In which case the operators $\tilde{P}_{t}:=P_{N-t}$, $t=0,1, \ldots, N$ satisfy

$\tilde{P}_{t}+\left(\mathcal{Q}+\mathcal{A}^{*} \tilde{P}_{t-1}\right) \mathscr{B} \mathcal{R}^{-1} \mathcal{B}^{*} \tilde{P}_{t}=\left(\mathcal{Q}+\mathcal{A}^{*} \tilde{P}_{t-1}\right) \mathcal{A}, \quad \tilde{P}_{0}=0$.

Suppose also that

$\|Q\|+\left\|\mathcal{A}^{*}\right\|\left\|\tilde{P}_{t-1}\right\|<1, \quad\left\|\mathscr{B} \mathcal{R}^{-1} \mathscr{B}^{*}\right\|<1$

then a unique bounded solution $\tilde{P}_{t}$ exists for (31) and also

$$
\begin{aligned}
\left\|\tilde{P}_{t}\right\| & \leq \frac{\left(\left\|\mathcal{Q}+\mathcal{A}^{*} \tilde{P}_{t-1}\right\|\right)\|\mathcal{A}\|}{\left(1-\left\|\mathcal{Q}+\mathcal{A}^{*} \tilde{P}_{t-1}\right\|\left\|\mathscr{B} \mathcal{R}^{-1} \mathscr{B}^{*}\right\|\right)} \\
& \leq \frac{\|\mathcal{A}\|}{\left(1-\left\|\mathscr{B} \mathcal{R}^{-1} \mathscr{B}^{*}\right\|\right)} .
\end{aligned}
$$

Hence, in order to guarantee that (31) has a solution for $t-1$ it is sufficient that $\left\|\left(\mathbb{Q}+\mathcal{A}^{*} \tilde{P}_{t}\right) \mathcal{B} \mathcal{R}^{-1} \mathscr{B}^{*}\right\|<1$ which (using the previous inequality) holds if $\|\mathscr{Q}\|+\|\mathscr{A}\|^{2}\left(1-\left\|\mathscr{B} \mathcal{R}^{-1} \mathscr{B}^{*}\right\|\right)^{-1}<1$. Moreover, $|\mathcal{A}|^{2} \leq \sum_{i=-\infty}^{\infty}\left|A_{i}\right|^{2}$ and $\|\mathcal{Q}\| \leq\|Q\|$. Combining these facts with the conditions given in the theorem guarantees the solvability of (31) for any $t=0,1, \ldots$.

Use of Theorem 3, the minimum value of the cost function for each fixed integer $N$ is $\left\langle\tilde{P}_{N} x_{0}, \mathcal{A} x_{0}\right\rangle$. Now let $N_{2}>N_{1}$. Hence for any admissible control $u$ and initial data $x \in l^{2}\left(\mathbb{R}^{n}\right)$

$$
\begin{aligned}
\sum_{t=1}^{N_{2}}\left[\left\langle\mathcal{Q} x_{t}, x_{t}\right\rangle+\left\langle\mathcal{R} u_{t}, u_{t}\right\rangle\right] & \geq \sum_{t=1}^{N_{1}}\left[\left\langle\mathcal{Q} x_{t}, x_{t}\right\rangle+\left\langle\mathcal{R} u_{t}, u_{t}\right\rangle\right] \\
& \geq \min _{u} J(u) \geq 0 .
\end{aligned}
$$

Hence, $\left\langle\tilde{P}_{N_{2}} x, \mathcal{A} x\right\rangle \geq\left\langle\tilde{P}_{N_{1}} x, \mathcal{A} x\right\rangle$ for any $x \in l^{2}\left(\mathbb{R}^{n}\right)$ and $N_{2}>N_{1}$.

Let $g_{\infty}(x)$ denote the minimum value of the cost function in (4)-(6) with initial data $x \in l^{2}\left(\mathbb{R}^{n}\right)$ and $N=\infty$. By analogy with (10), we can show that the optimal control in this case is given by

$u^{0}=-\left(\mathcal{R}+L^{*} \mathcal{Q} L\right)^{-1} L^{*} \mathcal{Q} w, \quad$ where $w=\left(x, \mathcal{A} x, \mathcal{A}^{2} x, \ldots\right)$.

Also it follows that $J_{\infty}(x)=\langle P w, w\rangle$, where $P$ is the linear operator given by

$P=Q-Q L\left(\mathcal{R}+L^{*} \mathcal{Q} L\right)^{-1} L^{*} \mathcal{Q} L$. 
Using (33) we have that for any $x \in l^{2}\left(\mathbb{R}^{n}\right)$

$0 \leq J_{\infty}(x)=\langle P w, w\rangle \leq\|P\|\langle w, w\rangle \leq C\langle x, x\rangle$

where the constant $C=1 /(1-\|\mathcal{A}\|)>0$. Also, for any integer $N$

$$
\begin{aligned}
J_{\infty}(x) & =\min _{u} J(u, x)=\sum_{t=1}^{\infty}\left[\left\langle\mathcal{Q} x_{t}^{0}, x_{t}^{0}\right\rangle+\left\langle\mathcal{R} u_{t}^{0}, u_{t}^{0}\right\rangle\right] \\
& \geq \sum_{t=1}^{N}\left[\left\langle\mathcal{Q} x_{t}^{0}, x_{t}^{0}\right\rangle+\left\langle\mathcal{R} u_{t}^{0}, u_{t}^{0}\right\rangle\right] \geq \min _{u} J(u)=\left\langle\tilde{P}_{N} x, \mathcal{A} x\right\rangle .
\end{aligned}
$$

Let $0 \leq N_{1}<N_{2}<\cdots$ be some increasing integer sequence. Then

$0 \leq\left\langle\tilde{P}_{N_{1}} x, \mathcal{A} x\right\rangle \leq\left\langle\tilde{P}_{N_{2}} x, \mathcal{A} x\right\rangle \leq \cdots \leq J_{\infty}(x) \leq C\langle x, x\rangle$

where the constant $C>0$ was given above. This means that $\left\{\mathcal{A}^{*} \tilde{P}_{N_{i}}\right\}$ is a nondecreasing bounded above sequence of nonnegative self-adjoint operators. Hence by the Banach-Steinhaus theorem this operator sequence has a strong nonnegative operator limit $\widetilde{T}$, i.e.

$\lim _{i \rightarrow \infty} \mathcal{A}^{*} \tilde{P}_{N_{i}} x=\widetilde{T} x \quad \forall x \in l^{2}\left(\mathbb{R}^{n}\right)$.

Since $r(\mathcal{A})<1$ then the operator $\mathcal{A}^{*}$ is invertible and from (34) it follows that the sequence $\tilde{P}_{N_{i}}$ is convergent. Let $\lim _{i \rightarrow \infty} \tilde{P}_{N_{i}} x=P x$ and also we have already shown that $J_{\infty}(x) \geq\left\langle\tilde{P}_{N} x, \mathcal{A} x\right\rangle$ for all $x \in l^{2}\left(\mathbb{R}^{n}\right)$ and any $N$. Taking limit as $N \rightarrow \infty$, we get $J_{\infty}(x) \geq$ $\langle P x, \mathcal{A} x\rangle$. Also it is easy to see that $J_{\infty}(x)$ takes the value $\langle P x, \mathcal{A} x\rangle$ when $u^{*}=-\mathcal{R}^{-1} \mathcal{B}^{*} P x$, i.e. $u=u^{*}$ is optimal. It is also easy to show that $u_{t}^{*}, t \in \mathbb{Z}_{+}$, produces the solution $x_{t}^{*}, t \in \mathbb{Z}_{+}$for

$x_{t+1}=\left(\mathscr{A}-\mathscr{B} \mathcal{R}^{-1} \mathscr{B}^{*} P\right) x_{t}, \quad x_{0}=x, \quad t \in \mathbb{Z}_{+}$.

Also this solution satisfies

$\left\langle P x_{t}^{*}, \mathcal{A} x_{t}^{*}\right\rangle-\left\langle P x_{t+1}^{*}, \mathcal{A} x_{t+1}^{*}\right\rangle=\left\langle Q x_{t+1}^{*}, x_{t+1}^{*}\right\rangle+\left\langle\mathcal{R} u_{t}^{*}, u_{t}^{*}\right\rangle$

and then

$$
\begin{aligned}
J\left(u^{*}\right) & =\sum_{t=0}^{\infty}\left[\left\langle\mathcal{Q} x_{t}^{*}, x_{t}^{*}\right\rangle+\left\langle\mathcal{R} u_{t}^{*}, u_{t}^{*}\right\rangle\right] \\
& =\langle P x, \mathcal{A} x\rangle+\lim _{t \rightarrow \infty}\left\langle P x_{t}^{*}, \mathcal{A} x_{t}^{*}\right\rangle .
\end{aligned}
$$

Since $x^{*} \in l_{2}^{2}\left(\mathbb{R}^{n}\right)$, then $\left\|x_{t}^{*}\right\| \rightarrow 0, t \rightarrow \infty$. This shows that $J_{\infty}(x)=J\left(u^{*}\right)=\langle P x, \mathcal{A} x\rangle$ and the proof is complete.

The optimal solution for the problem (4)-(6) can be re-formulated in the frequency domain using the discrete Fourier transform. (These results are of interest in engineering, where the frequency domain is a standard extremely important option.)

Theorem 5. The discrete Fourier transform

$u_{t}(\omega)=\sum_{m=-\infty}^{\infty} u^{0}(t, m) \mathrm{e}^{-j m \omega}, \quad \omega \in[0,2 \pi], j^{2}=-1$

of the optimal control $u^{0}(t, m)$ (with $T \rightarrow \infty$ ) can be written as

$u_{t}(\omega)=K(\omega) X_{t}(\omega)$

where $X_{t}(\omega)$ denotes the Fourier transformation of the optimal trajectory $x^{0}(t, m)$ and

$K(\omega)=-\left[R+B^{*} P(\omega) B\right]^{-1} B^{*} P(\omega) A(\omega), \quad A(\omega)=\sum_{k=-\infty}^{+\infty} \mathrm{e}^{j k \omega} A_{k}$.

Here $P(\omega), \omega \in[0,2 \pi]$ is given by

$P(\omega)=Q+A^{*}(\omega) P(\omega) A(\omega)$

$$
-A^{*}(\omega) P(\omega) B\left[R+B^{*} P(\omega) B\right]^{-1} B^{*} P(\omega) A(\omega) .
$$

Also, the minimal cost value is

$J\left(u^{0}\right)=\frac{1}{2 \pi} \int_{0}^{2 \pi}\left\langle X_{0}(\omega), P(\omega) X_{0}(\omega)\right\rangle \mathrm{d} \omega$.

Proof. Applying the discrete Fourier transformation to (4) with respect to the variable $m$, i.e.

$X_{t}(\omega)=\sum_{m \in \mathbb{Z}} x^{0}(t, m) \mathrm{e}^{-j m \omega}, \quad \omega \in[0,2 \pi]$

gives

$X_{t+1}(\omega)=A(\omega) X_{t}(\omega)+B U_{t}(\omega)$,

$A(\omega)=\sum_{k=-\infty}^{\infty} \mathrm{e}^{i k \omega} A_{k}, \omega \in[0,2 \pi]$

Using Parseval's identity, the cost function can be written as

$J(u)=\frac{1}{2 \pi} \sum_{t \in Z_{+}} \int_{0}^{2 \pi}\left\langle X_{t}(\omega), Q X_{t}(\omega) X_{T}(\omega)\right\rangle+\left\langle U_{t}(\omega), R U_{t}(\omega)\right\rangle \mathrm{d} \omega$.

Let $P(\omega), \omega \in[0,2 \pi]$, be an arbitrary collection of nonnegative operators from $\mathbb{C}^{n}$ to $\mathbb{C}^{n}$ such that $\int_{0}^{2 \pi}\|P(\omega)\| \mathrm{d} \omega<\infty$. Then

$$
\begin{aligned}
0= & \left\langle P(\omega) X_{0}(\omega), X_{0}(\omega)\right\rangle-\sum_{t \in Z_{+}}\left\langle P(\omega) X_{t}(\omega), X_{t}(\omega)\right\rangle \\
& +\sum_{t \in Z_{+}}\left\langle P(\omega) A(\omega) P(\omega) X_{t}(\omega)\right. \\
& \left.+B U_{t}(\omega), A(\omega) P(\omega) X_{t}(\omega)+B U_{t}(\omega)\right\rangle .
\end{aligned}
$$

Integrating this last identity over $\omega \in[0,2 \pi]$, adding the result to $J$, and then adding and subtracting

$\left\langle P(\omega) A(\omega) X_{t}(\omega), B\left[R+B^{*} P(\omega) B\right]^{-1} B^{*} P(\omega) A(\omega) X_{t}(\omega)\right\rangle$

from the result gives

$$
\begin{aligned}
J(u)= & \frac{1}{2 \pi} \int_{0}^{2 \pi}\left\langle P(\omega) X_{0}(\omega), X_{0}(\omega)\right\rangle+\sum_{t \in Z_{+}}\left[\left\langle F(\omega) X_{t}(\omega), X_{t}(\omega)\right\rangle\right. \\
& \left.+\left\langle\left(R+B^{*} P(\omega) B\right) V_{t}(\omega), V_{t}(\omega)\right\rangle\right] \mathrm{d} \omega
\end{aligned}
$$

where

$$
\begin{aligned}
F(\omega)= & Q-P(\omega)+A^{*}(\omega) P(\omega) A(\omega) \\
& -A^{*}(\omega) P(\omega) B\left[R+B^{*} P(\omega) B\right]^{-1} B^{\mathrm{T}} P(\omega) A(\omega) \\
V_{t}(\omega)= & U_{t}(\omega)+\left[R+B^{*} P(\omega) B\right]^{-1} B^{*} P(\omega) A(\omega) X_{t}(\omega) .
\end{aligned}
$$

Note that the inverse of the operators here exist because $P(\omega) \geq 0$ and $R>0$ is positive operators.

The second term in the cost function here does not depend on control input since $X_{0}(\omega)=\sum_{s \in \mathbb{Z}} \varphi(s) \mathrm{e}^{-i s \omega}, \omega \in[0,2 \pi]$. Choose now $P(\omega)$ such that $F(\omega)=0$ holds. Then the cost function can be rewritten as

$$
\begin{aligned}
J(u)= & \frac{1}{2 \pi} \int_{0}^{2 \pi}\left\langle P(\omega) X_{0}(\omega), X_{0}(\omega)\right\rangle \\
& +\sum_{t=0}^{+\infty}\left\langle\left[R+B^{*} P(\omega) B\right]^{-1} V_{t}(\omega), V_{t}(\omega)\right\rangle \mathrm{d} \omega
\end{aligned}
$$

and clearly its minimum value is

$J\left(u^{0}\right)=\frac{1}{2 \pi} \int_{0}^{2 \pi}\left[\left\langle P(\omega) X_{0}(\omega), X_{0}(\omega)\right\rangle\right] \mathrm{d} \omega$

which is feasible if, and only if, $V_{t}(\omega)=0$, i.e. if, and only if, $U_{t}(\omega)=K(\omega) X_{t}(\omega)$. Thus the required representation for the optimal control law and the function $K(\omega)$ and $P(\omega)$ have been obtained and the proof is complete. 
The following result holds for the feedback control case.

Theorem 6. The optimal feedback control for (4), (5) and (6) in the case when $T \rightarrow \infty$ is given by

$u(t, m)=\sum_{i=-\infty}^{+\infty} K_{i} x(t, m+i)$

where $K_{i}, i \in \mathbb{Z}$, is a set of $r \times n$-matrices.

Proof. First note that (37) admits the solution $P=P(z)$ which is analytic in a domain including the unit disc of complex plane $\mathbb{C}$ and hence the function $K=K(z)$ is analytic in the same domain. Hence $\exists \epsilon>0$ such that $K(z)$ can be expanded in series form as

$K(z)=\sum_{i=-\infty}^{+\infty} K_{i} z^{i}, \quad 1-\epsilon<|z|<1+\epsilon$

and also

$U_{t}(\omega)=K(\omega) X_{t}(\omega)=\sum_{i=-\infty}^{+\infty} K_{i} \mathrm{e}^{i j \omega} X_{t}(\omega)$.

The inverse Fourier transform of $X_{t}(\omega)$ now yields

$$
\begin{aligned}
u^{0}(t, m) & =\frac{1}{2 \pi} \int_{-\pi}^{\pi} \sum_{i=-\infty}^{+\infty} K_{i} \mathrm{e}^{i j w} X_{t}(w) \mathrm{e}^{j m w} \mathrm{~d} w \\
& =\frac{1}{2 \pi} \sum_{i=-\infty}^{+\infty} K_{i} \int_{-\pi}^{\pi} X_{t}(w) \mathrm{e}^{j(m+i) w} \mathrm{~d} w=\sum_{i=-\infty}^{+\infty} K_{i} x(t, m+i) .
\end{aligned}
$$

Note now that the matrices $K_{i}$ are the coefficients of the series expansion of $K(z)$ and the proof is complete.

\section{Conclusions}

This paper deals with the so-called wave repetitive processes whose existence and relevance to engineering applications has been highlighted. These processes evolve in the upper-half of the 2D plane and hence existing control systems' analysis tools for repetitive processes which evolve in the positive quadrant of the 2D plane is not applicable. Consequently, as the first major analysis tool for this new model, an optimal control problem has been formulated and solved. This is based on first introducing a 1D equivalent model of the process dynamics in an infinitedimensional systems' setting. Also it has been shown that this solution can be written in the feedback form. These results provide a solid basis on which to progress to the design and implementation of the control laws.

\section{Acknowledgement}

The first author's work was partially supported by the Research Foundation of Belarus.

\section{References}

[1] E. Rogers, K. Galkowski, D.H. Owens, Control Systems Theory and Applications for Linear Repetitive Processes, in: Lecture Notes in Control and Information Sciences Series, vol. 349, Springer Verlag, Berlin, 2007.

[2] H. Zhao, Passive, iterative and repetitive control for flexible distributed parameter systems, Ph.D. Thesis, Department of Mechanical and Nuclear Engineering, The Pennsylvania State University, USA, 2005.

[3] M. Bisiacco, New results in 2D optimal control theory, Multidimensional Systems and Signal Processing 6 (1995) 189-222.

[4] H. Kwakernaak, R. Sivan, Linear Optimal Control Systems, Willey, New York, 1972. 